\title{
Integration of Multispectral Images from Modern Earth Remote Sensing Systems
}

\author{
A. Kuznetcov, P. Svetelkin \\ Ryazan State Radio Engineering University, Gagarin Str., 59/1, Ryazan, Russia, 390005
}

Keywords: pansharpening, moving objects elimination, true color images

\begin{abstract}
This paper shows algorithms of color image acquisition by the use of a panchromatic high resolution image and multispectral lower resolution images. The algorithm of artifact elimination from moving and high objects was developed. The algorithm of color image synthesis in true colors was developed on the basis of the NDVI index.
\end{abstract}

\section{Introduction}

Modern earth remote sensing systems such as "Resource-DK", "Resource-P" and "Canopus-V" use scanners composed of several optoelectronic devices (OED) in order to obtain multispectral images of the earth surface. OED are located in the focal plane sequentially one after another. Therefore panchromatic and multispectral images are formed with a short time delay relative to each other. These formed images are characterized by a set of features. Firstly panchromatic images have higher spatial resolution than multispectral ones. Secondly images are geometrically distorted relative to each other. Taking into account above mentioned features, the tasks of multispectral image integration are the following:

1) high-precision geometrical stitching of images obtained in different moments in order to receive video data arrays which will be used for the following processing;

2) synthesis of multispectral images with high spatial resolution according to the data of panchromatic and multispectral imagery;

3) formation of high-quality color synthesized images of the earth surface which will be used for visual decoding.

This paper considers algorithms of above mentioned task solutions.

\section{Algorithms of the Image Integration}

Algorithm of the Multispectral Images Geometrical Stitching. Algorithms of geometrical stitching of images obtained in different moments include two stages [1]. At the first stage equations of geometrical correspondence between basic and stitched multispectral images for the medium terrain altitude are set up. Then all images are transformed into the basic image coordinate system. The equation of geometrical correspondence is the following:

$$
\begin{gathered}
m_{\lambda}=F_{\lambda}^{-1}\left[F_{c}\left(m_{c}, n_{c}\right), \Phi_{c}\left(m_{c}, n_{c}\right)\right], \\
n_{\lambda}=\Phi_{\lambda}^{-1}\left[F_{c}\left(m_{c}, n_{c}\right), \Phi_{c}\left(m_{c}, n_{c}\right)\right], \lambda=1,2, \ldots, \lambda \neq c,
\end{gathered}
$$

where $\left(m_{\lambda}, n_{\lambda}\right)$ and $\left(m_{c}, n_{c}\right)$ - line and column numbers of stitched and basic images with number $c$; $F_{c}(),. \Phi_{c}($.$) - equations allowed calculating geodesic coordinates, latitude and longitude of a pixel$ with number $(m, n) ; F_{\lambda}^{-1}(\cdot), \Phi_{\lambda}^{-1}(\cdot)$ - mathematics allowed online determining planar coordinates of a pixel by geodesic coordinates.

At the second stage residual geometrical mismatches caused by the relief and altitude objects are removed. To this effect, planes of stitching images are divided into triangular segments with the required level of thickening. 
Multispectral images obtained within the geometrical stitching correspond to the processing level 1 established in the international practice and are used for the following transformation into cartographic projections.

\subsection{Algorithm of the panchromatic and multispectral image integration}

Integration of the data obtained by means of the panchromatic and multispectral imagery is called "pansharpening" in the world practice. The challenge is to provide spatial resolution of the synthesized image $D_{\gamma}=\left\{d_{\gamma}\left(m_{c}, n_{c}\right)\right\}, m_{c}=\overline{1, M}, n_{c}=\overline{1, N}$ and spectral characteristics of the multispectral image.

One of the known approaches to form the multispectral component is a modulation algorithm according to which the desired constituent $d_{\gamma}$ is calculated as

$$
d_{\gamma}=\frac{3 b_{\gamma}}{\sum_{\gamma} b_{\gamma}} b_{p}, \quad \gamma \in\{b, g, r\},
$$

where $b_{p}, b_{\gamma}$ - pixel brightness of the panchromatic and multispectral images.

Experimental researches have shown that this modulation algorithm does not lead to the loss of spatial resolution of a color synthesized image. However as it is shown in Figure 1b, the color palette of a new image differs from the initial multispectral image.

The known coloring algorithm HCS [2] saves a color palette of initial multispectral images. However there is a loss of resolution of the obtained color synthesized image in relation to a panchromatic image with high resolution.

According to the algorithm IHS [3, 4], the resultant image is formed by the following expressions:

$$
i=\sum_{\gamma} \alpha_{\gamma} b_{\gamma}, \quad \widehat{b}_{p}=\bar{i}+\frac{\sigma_{I}}{\sigma_{P}}\left(b_{p}-\overline{b_{p}}\right), \quad d_{\gamma}=b_{\gamma}+\left(\widehat{b}_{p}-i\right),
$$

where $\bar{i}-$ a mean value of brightness of pixels of the image $I=\left\{i\left(m_{c}, n_{c}\right)\right\}, \widehat{b}_{p}$ - aligned brightness of a pixel of the panchromatic image; $\bar{i}, \overline{b_{p}}, \sigma_{I}, \sigma_{p}$ - mean values and mean square deviations of images $I$ and $B_{p}$. Disadvantages of this algorithm are color distortions and also the necessity to choose the coefficient $\alpha_{i}$.

The integration result of higher quality is obtained using the expression [5]:

$$
d_{\gamma}=b_{\gamma} \frac{b_{p}}{b_{\tilde{p}}}, B_{\tilde{p}}=B_{p} \otimes h_{7 \times 7},
$$

where $\otimes-$ convolution with a bleeding mask of $h$ dimensions of $7 \times 7$ elements.

However increase of a noise term of the resultant image limits practical use of such approach.

This paper suggests an algorithm free of mentioned shortcomings and allowed saving spatial resolution of a panchromatic image and initial colors of multispectral images with lower resolution. The algorithm idea is based on extraction of high-frequency constituents from a panchromatic image and their introduction into a multispectral image. The resultant color image is formed by the expression:

$$
d_{\gamma}=b_{\gamma}+\widehat{b}_{p}, \hat{b}_{p}=\frac{\sigma_{I}}{\sigma_{P}}\left(b_{p}-b_{\tilde{p}}\right), B_{\tilde{p}}=B_{p} \otimes h_{7 \times 7} .
$$

Besides the obtained image $\widehat{B}_{p}$ on areas with short brightness jumps has null brightness codes that does not lead to the colour distortion of such objects. The example of the algorithm operation is shown in Figure 1c. 


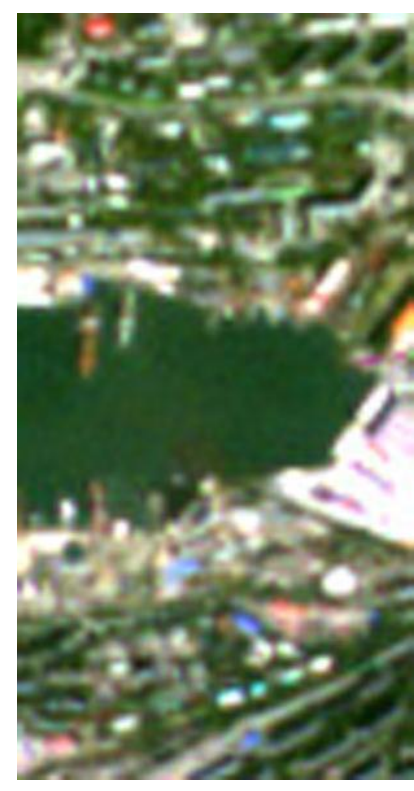

a

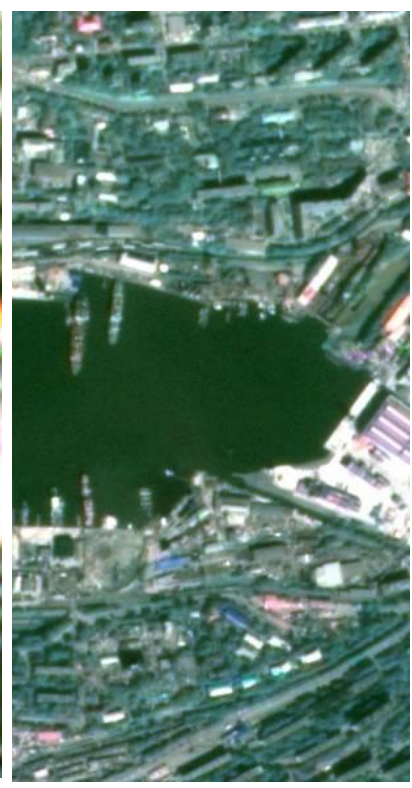

$\mathrm{b}$

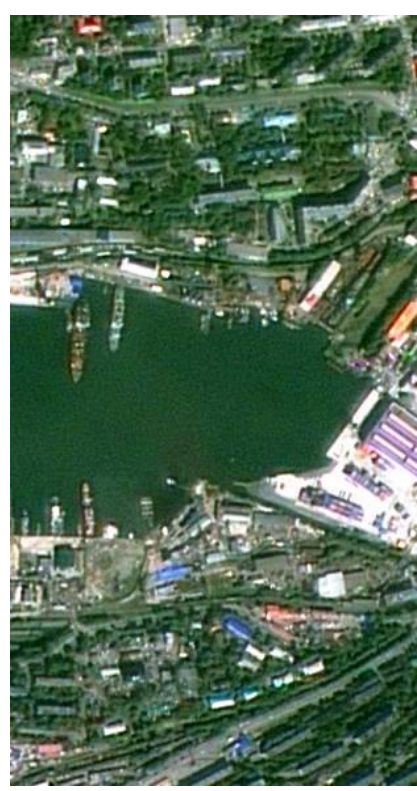

C

Fig. 1: Examples of the synthesis of color images obtained from the space complex "Canopus-V" a - image from a sensor MSS; $\mathrm{b}$ - color image obtained by the modulation algorithm; $\mathrm{c}$ - color image obtained by the modified algorithm IHS.

\subsection{Algorithm of the high quality color image formation}

The following two tasks are solved under formation of color synthesized images of the earth surface: elimination of defects caused by moving objects and formation of the earth surface image in true colors.

The highly productive algorithm of color defect compensation appeared on the RGB-image in the form of three color copies of a moving object consists in the following:

Color RGB_components are transferred into the coordinate system similar to $Y C_{b} C_{r}$ where $Y-\mathrm{a}$ brightness component, $C_{b}$ and $C_{r}$ - chromatic blue and chromatic red components. The transfer is executed by the expression

$$
\left(\begin{array}{c}
y \\
c_{b} \\
c_{r}
\end{array}\right)=\left(\begin{array}{ccc}
0 & 1 & 0 \\
0,1687 & -0,3313 & 0,5 \\
0,5 & -0,4187 & 0,0813
\end{array}\right)\left(\begin{array}{c}
\hat{b}_{r} \\
b_{g} \\
\hat{b}_{b}
\end{array}\right) .
$$

Then color components are formed with low spatial resolution:

$$
C_{b}^{*}=C_{b} \otimes h_{j \times j}, C_{r}^{*}=C_{r} \otimes h_{j \times j} .
$$

Then components of the color synthesized image $D$ are calculated by the expression:

$$
\left(\begin{array}{l}
d_{r} \\
d_{g} \\
d_{b}
\end{array}\right)=\left(\begin{array}{ccc}
0,7721 & -0,3441 & 2,1162 \\
1 & 0 & 0 \\
0,3908 & 2,056 & -0,6938
\end{array}\right)\left(\begin{array}{c}
y \\
c_{b}^{*} \\
c_{r}^{*}
\end{array}\right) .
$$

The example of the algorithm operation is shown in Figure 2. 


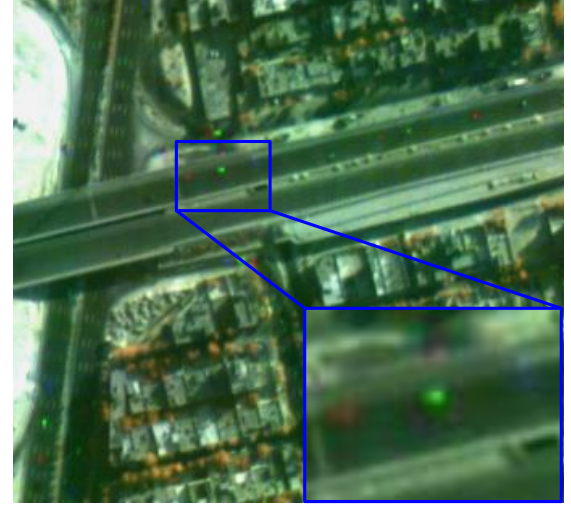

a

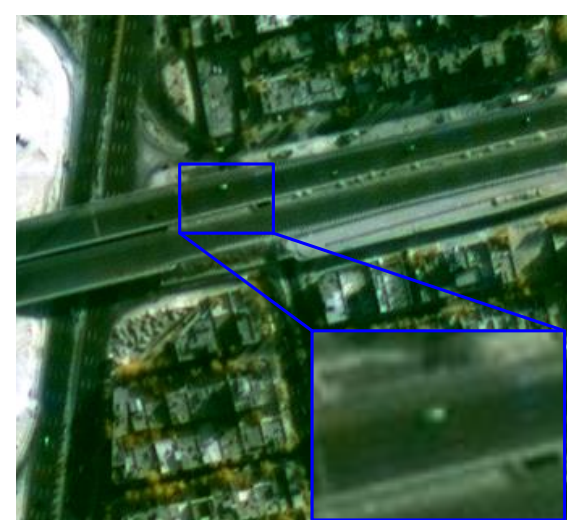

b

Fig. 2: Example of elimination of moving objects under RPB-imagery: $\mathrm{a}$ - initial image, $\mathrm{b}$ - image obtained using the modulation algorithm

A lot of remote sensing systems obtain the earth surface image in spectral ranges which differ from the natural representation. Images obtained from such systems represent green plants with tints of red. So it is necessary to develop an algorithm of the true color image synthesis. Let's analyze known algorithms of such image representation.

The description of TerraLook [6] service indicates that images are represented in true colors with a synthesized blue channel:

$$
d_{r}=b_{r}, \quad d_{g}=\frac{2}{3} b_{g}+\frac{1}{3} b_{i r}, \quad d_{b}=\frac{2}{3} b_{g}-\frac{1}{3} b_{i r} .
$$

where $« i r »-$ a sign of the near infrared imagery range.

The first three imagery ranges of the ASTER equipment are similar to imagery channels of the space complex "Resource-DK" that is why the mentioned algorithm applied to images of "Resource-DK" provides the results shown in Figure 3b. It should be noted that some images have unnatural colors (Figure 3f). The analogous algorithm different in coefficients is used for SPOT images:

$$
d_{r}=b_{r}, \quad d_{g}=\frac{3}{4} b_{g}+\frac{1}{4} b_{i r}, \quad d_{b}=\frac{3}{4} b_{g}-\frac{1}{4} b_{i r} .
$$

Its application provides the results similar to the results of the TerraLook algorithm (Figure $3 \mathrm{c}, \mathrm{g}$ ). The disadvantages of algorithms SPOT and TerraLook are coloration of shades from high objects in blue color and unnatural blueing of water objects.

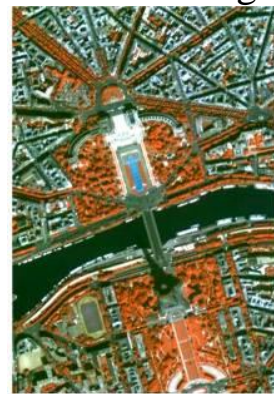

a

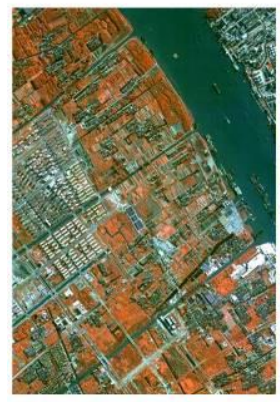

$\mathrm{e}$

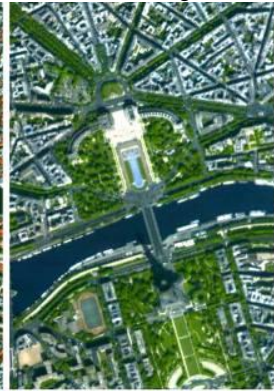

b

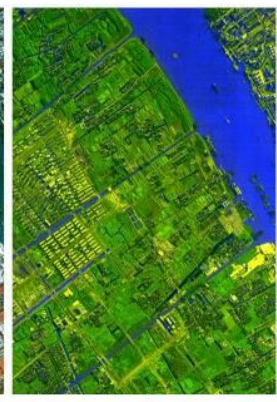

f

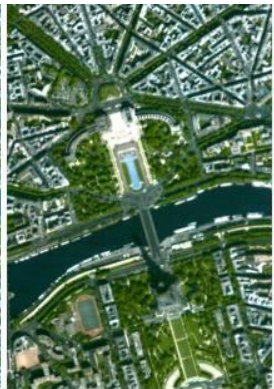

c

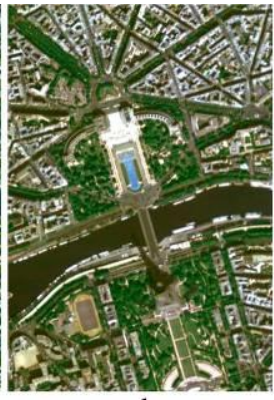

d

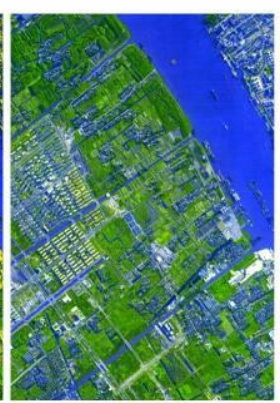

$\mathrm{g}$

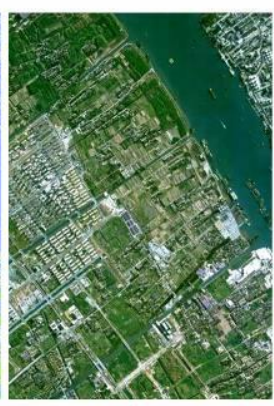

$\mathrm{h}$ 
Fig. 3: Examples of the color image synthesis: a, e - initial images; $b, f-$ images synthesized according to the TerraLook algorithm; c, g - images synthesized according to the SPOT algorithm; d, $\mathrm{h}$ - color images synthesized according to the developed algorithm using the NDVI index.

Testing of known algorithms has shown that they are not suitable for creation of color image in the color close to natural. So the following approach is suggested. It is possible to calculate a vegetation index i.e. to select green photosynthetic plans on the image on the basis of the image brightness of the second and third channels. The most widespread vegetation index NDVI is calculated by the expression

$$
K_{N D V I}=\frac{b_{i r}-b_{r}}{b_{i r}+b_{r}} .
$$

Besides the vegetation index $K_{N D V I}$ possesses the value in the range $[-1 ; 1]$, and for green photosynthetic plans $K_{N D V I}>0$.

The pixel brightness is normalized in order to calculate the vegetation index. Then the expression (11) has the form

$$
K_{N D V I}=\frac{K_{\text {omt }} b_{i r}-b_{r}}{K_{\text {omt }} b_{i r}+b_{r}}, K_{\text {omH }}=\frac{\bar{b}_{r}}{\bar{b}_{i r}},
$$

where $\bar{b}_{r}, \bar{b}_{i r}$ - mean values of pixel brightness of images of blue and red infrared channels. So, for example, for images obtained from the space complex "Resource-DK" the vegetation index value does not exceed 0,3 , and then the empirical function is introduced

$$
f_{H}\left(b_{r}, b_{i r}\right)=\left\{\begin{array}{l}
3 K_{N D V I} \bar{b}_{i r}, K_{N D V I}>0, \\
0, K_{N D V I} \leq 0 .
\end{array}\right.
$$

Then the pixel brightness of the color synthesized image $D$ in colours close to natural is calculated by the expressions:

$$
d_{b}=b_{g}, \quad d_{g}=b_{r}, \quad d_{r}=b_{i r}-f_{H}\left(b_{r}, b_{i r}\right)
$$

for GRIr imagery mode,

$$
d_{b}=b_{g}, \quad d_{g}=b_{p}-b_{i r}, \quad d_{r}=b_{i r}-f_{H}\left(b_{p}, b_{i r}\right)
$$

for GPIr imagery mode and

$$
d_{b}=b_{g}, \quad d_{g}=b_{r}, \quad d_{r}=b_{p}-b_{r}-f_{H}\left(b_{r}, b_{p}\right)
$$

for GRP imagery mode.

The color image obtained on the basis of expression (14) is shown in Figure 3d. It shows that the color of green plants became more natural and the color of urban areas did not change.

So the suggested algorithm is stable to fluctuations of spectral ranges and allows obtaining color images in true colors on the basis of video data from obtained such space complexes as "ResourceDK", "Meteor -M" and others.

\section{Conclusions}

The algorithms stated in the paper are accompanied by examples. The practical use of suggested algorithms in software data processing complexes from Russian space remote sensing systems "Resource-DK", "Resource-P", "Canopus" and "Meteor-M" confirms the effectiveness of suggested technical solutions.

\section{References}

[1] V. Eremeev, A. Kuznetcov, G. Miatov, O. Presnyakov, V. Poshekhonov and P. Svetelkin "Image structure restoration from sputnik with multi-matrix scanners", Proc. SPIE 9244, Image and Signal Processing for Remote Sensing XX, 92440F (October 15, 2014); doi:10.1117/12.2066631; http://dx.doi.org/ 10.1117/12.2066631 
[2] WorldView-2 Pan-Sharpening. C. Padwick, M. Deskevich, F. Pacifici, S. Smallwood. URL: http://www.digitalglobe.com/downloads/WorldView-2_Pan-Sharpening.pdf

[3] S Rahmani, M Strait, D Merkurjev, M Moeller, T Wittman, An adaptive HIS pan-sharpening method. IEEE Geoscience And Remote Sensing Letters 7(3), 746-750 (2010)

[4] M. Choi, "A new intensity-hue-saturation fusion approach to image fusion with a tradeoff parameter,” IEEE Trans. Geosci. Remote Sens., vol. 44, no. 6, pp. 1672-1682, Jun. 2006.

[5] Pleiades Imagery User Guide. October 2012 - V 2.0

[6] TerraLook: бесплатные космические снимки ASTER, TM, ETM+. URL: http:/gislab.info/qa/terralook.html

[7] Interpreting Optical Remote Sensing Images. URL: http://www.crisp.nus.edu.sg/ research/tutorial/opt_int.htm 